\title{
USD: Scalable Bandwidth Allocation for the Internet
}

\author{
Zheng Wang \\ Bell Laboratories, Lucent Technologies \\ 101 Crawfords Corner Road, Holmdel, NJ 07733 \\ zhwang@dnrc.bell-labs.com
}

\begin{abstract}
In this paper, we present a differentiated service scheme called "User-Share Differentiation (USD)". The USD scheme is designed for long-term bandwidth allocation without per-session signaling. The scheme allows ISPs to provide traffic isolation on a per-user basis and guarantee proportional fairness. We first look at the background for differentiated services, and the problems with the current proposals. We then present the details of the USD scheme and examine the implementation and deployment issues.
\end{abstract}

\section{Keywords}

Quality of services, differentiated service, weighted fair queuing, bandwidth allocation, proportional fairness

\section{INTRODUCTION}

The current Internet is built on the best-effort model where all packets are treated as independent datagrams and are serviced on the FIFO basis. The best effort model does not provide any form of traffic isolation inside the network and the network resources are completely shared by all users. As a result, the Internet suffers from the "Problem of Commons" where greedy users try to grab as much resource as possible. Such a system can become unstable and lead to congestion collapse. The Internet currently still works because most end systems use TCP congestion control mechanisms and back off during congestion. However, such dependence on the end systems' cooperation is increasingly becoming unrealistic. Inevitably, people start to exploit the weakness of the best effort model to gain more resources. An example of this is to establish multiple TCP connections in web browsers to gain greater share of the bandwidth. The best effort model also prevents ISPs from meeting the different needs of their customers since it is difficult to allocate more resources to those who are willing to pay more.

The original version of this chapter was revised: The copyright line was incorrect. This has been corrected. The Erratum to this chapter is available at DOI: $10.1007 / 978-0-387-35388-3 \_42$ 
The problems with the best effort model have been long recognized. For the last a couple of years, QoS provision has been one of the hottest areas in networking research, and various aspects of the issue have been extensively studied including traffic analysis, admission control, resource reservation, scheduling, QoS routing, and operating system support. The architectures of various proposed solutions differ in details. Nevertheless, the underlying model is rather similar. Essentially, applications make resource reservation on an end-to-end per session basis. We refer to this model as the End-to-End Per Session (EEPS) model. In the Internet community, RSVP and INT-SERV are examples of protocols and service models based on this model (Zhang et al 1993). In general, the EEPS model achieves QoS guarantees through the following steps:

- The application characterizes its traffic, and describes its requirements in a flow specification.

- The QoS routing figures out one or more candidate paths based on the requirements.

- A reservation or signaling protocol then checks for admission control hop-byhop and installs the reservation over the candidate path if there is sufficient resources.

- The schedulers enforced the reservation for each flow.

The past work based on the EEPS model has given us valuable insights and practical experience with resource allocation in the Internet. However, the EEPS model has a number of problems:

- On-demand per-session reservation does not work well for Web-based applications. For applications with long lasting sessions such as video conferencing, the delay and overheads of reservation is minimal. But for transaction-based applications such as Web, where a user can go through many destinations in a few seconds, setting up a reservation for each transaction has a high overhead. Furthermore, the resource requirement for Web traffic is usually difficult to determine. Very often, a user does not know how big an object is before fetching it. Also, delay variation affects Webbased application far less drastically compared with applications like video conferencing, thus there is more space for adaptation.

- Security, accounting and administrative support represent a significant amount of overheads in resource reservation. As each reservation is a service contract between the user and ISPs along the path, resource reservation goes far beyond simply installing state inside the network. Each request has to be authenticated and the user's account to be charged. Within the user organization, there may be internal procedures for approval and coordination of requests from individual users. All those have two implications. First, accounting and administrative support has to be an integrated part of a resource reservation system before the system can be deployed. Second, there is a need for aggregated reservation in order to reduce overheads of resource reservation for short-lived sessions. 
- The inter-ISP settlement is a complex issue and is unlikely to be resolved in the near term. When a path traverses multiple ISPs, an end-to-end reservation requires an agreement among all major ISPs on the inter-ISP settlement. Any single ISP that does not participate in such an agreement may break the reservation. Incremental deployment measures are essentially for the success of any reservation systems.

- The fine granularity of the EEPS model can lead to some scalability problems as the number of reservations increase. The classifier has to check the five fields (source address, destination address, source port, destination port and protocol) to determine if a packet belongs to one of the reserved flows. Such fine granularity lookup can be expensive when the number of flows is large. When the sessions are short-lived, the control messages can also be substantial and the processing of the control messages may become the bottleneck.

At the time when the work on the EEPS model started a couple of years back, realtime applications such as video conferencing were regarded as the mainstream application for the future Internet. For such applications, the on-demand persession reservation makes sense. However, the advent of the Web has changed the landscape significantly. The majority of the Internet traffic today is web-based and tends to be short-lived and transaction-oriented.

In the recent months, the term "differentiated services" has been used to describe new service models and mechanisms to achieve bandwidth allocation for aggregated traffic without per-session reservation. The basic requirements for such new service models and mechanisms are as follows:

- Aggregated bandwidth allocation without the need for per-session signalling.

- Long-term service contracts within a single domain.

- Integrated and simplified accounting.

- Better traffic isolation for performance predictability.

- Better services to users who are willing to pay more.

In this paper, we present a scalable differentiated service scheme called "UserShare Differentiation (USD)" (Wang 1997). The USD scheme is designed for long-term bandwidth allocation without per-session signaling. The scheme allows ISPs to provide traffic isolation on a per-user basis and guarantee proportional fairness. We first look at the background for differentiated services, and the problems with the current proposals. We then present the details of the USD scheme and examine the implementation and deployment issues.

\section{RELATED PROPOSALS}

In this section, we examine two related proposals that have been put forward for differentiated services. 


\section{Premium Service}

The premium service model described by Nichols et al (1997) provides guaranteed peak-rate bandwidth for aggregated traffic flows from users at the ISP entry points. The proposal creates a "premium" service that is provisioned according to the worst-case requirements and guaranteed by priority queuing. Routers at the edges of the network filter packets and set the premium bit in the packet header according to users' premium bandwidth profile. Inside the network, packets with the premium bit set are transmitted prior to the best effort packets.

The premium service proposal represents an extreme form of resource allocation, where the network capacity is effectively reduced by the amount allocated to the premium class traffic and the best effort traffic suffers all the consequences of congestion. The premium class requires strict admission control at the entry points to the ISP. Users' traffic is shaped to the allocated bandwidth. The arriving packets that exceed the allocated bandwidth profile are either delayed or dropped.

\section{Assured Service}

The assured service defined in the profile-based tagging scheme uses drop priority to differentiate traffic (Clark and Wroclawski 1997). Each user is assigned with a service profile that describes the "expected capacity" from the ISP. The traffic from a user is checked by a profile meter at the entry points to the ISP. Packets that are out of the profile can still go through but they are tagged as such by the profile meter. When congestion occurs inside the network, the routers drop the tagged packets first. When traffic is complaint to the agreed profile, it is expected that a user can have predictable level of services.

The premium service proposal and the profile-based tagging proposal are similar in that both proposals create an "upper" class and give preference to the upper class over the best effort class. In both proposals, the classification of packets is done at the edges and the class information is encoded in the packet header. The difference lies in that the premium service drops packets that are out-of-profile at the entry points to the ISP while the assured service still allows such packets go through in the hope that they may get to their destinations.

The two proposals attempt to push all policy-related processing to the edges of the network, and inside the core, the routers just forward packets based on the ToS bits in the header. While this approach has the advantage of simplicity, there are also a number of problems:

- Provisioning. Both proposals attempt to provide guaranteed bandwidth to the upper class traffic through admission control at the edges of the network. The assumption here is that with admission control around the edges and proper provisioning of the network, one can effectively eliminates congestion for the upper class. However, given a set of upper class users, the problem of dimensioning the network to meet the bandwidth guarantees to upper class users is a non-trivial problem. Since traffic flows are dynamic; any source can generate traffic to different destinations at different rates and the routes to the destinations may also change. Thus it is difficult for the edges to have the knowledge of traffic distribution inside the network. To provide any sort of 
guarantees, it is necessary to provision the network for the worst-case where the one assume that all upper class traffic may go through the weakest link in the network.

- Choosing profile. Choosing a proper profile for a user is not a straightforward task either. It can be viewed as the reverse problem of provisioning. For a given network, how can one decide the best profile users can be assigned to. Note that a profile applies to the aggregated traffic flow from a user organization going through the entry point to its ISP. When a network does not have uniform bandwidth provisioning, profiles are likely to be destinationspecific. For example, suppose that an ISP has a link to a neighbor ISP with a capacity $600 \mathrm{Mbps}$ and a link to the Internet backbone with a capacity of 100 Mbps. If a user is communicating with another user in the neighbor ISP, the user can have a rate limit of $6 \mathrm{Mbps}$ but only $1 \mathrm{Mbps}$ if the user's traffic goes through the backbone access link. In this case, a profile of either $6 \mathrm{Mbps}$ or 1 Mbps is not appropriate for the user. When a user is sending traffic to multiple destinations, the situation becomes even more complicated.

- Reverse traffic. Both the premium service and assured service proposals largely focus on the case where the users send packets towards the ISP. The bits in the packet header are set at the entry points to the ISP before mixed with packets from other sources, therefore the profile meter only need to know the admission control policy for the sender. However, in many cases, the users actually pulling the traffic in from the ISP. A typical example of this is Webbased applications which usually retrieve information from the Web servers. To apply the premium service and assured service models to such reverse traffic, the premium/assured bits have to be set at the server side or at the ISPISP boundary. There are a number of problems. First, it implies that the profile meter at an ISP-ISP boundary has to know the admission control policy for all its users. Second, if there may be multiple ISP-ISP boundaries, it becomes necessary for the profile meters at all boundaries to cooperate in order to make sure the sum of all upper class traffic for a user matches its profile.

- Starvation. With premium class scheme, that the congestion is invisible to the premium class, the network will no longer to provide any congestion signal for the premium class traffic. For TCP flows in the premium class, the sender's window will grow to the point that all bandwidth allocated to the premium class is taken up. If the bandwidth provisioning for the premium class is not done with care, best effort traffic will see significant degradation and may be starved completely.

- The profile-based tagging only provides limited protection against misbehaving sources. Since in profile-based tagging, the network deals with the tagged packets in a FIFO fashion. A misbehaving source can still gain more bandwidth by injecting excessive traffic. The problem can be aggravated when the fixed profiles are significantly over (or below) the level appropriate for the congested links. In such a case, the majority of the packets are not tagged (or tagged). Thus the tagging pro-vides little information to 
enforce differentiation, and network behavior will be close to simple FIFO best effort.

\section{USER-SHARE DIFFERENTIATION}

In this section, we present User-Share Differentiation (USD), a scalable bandwidth allocation scheme for differentiated services, and discuss the key design principles behind the scheme.

\subsection{Overview}

The USD scheme is designed to provide long-term bandwidth allocation for aggregated traffic flows. It differs from the premium service and assured service in a number of ways:

- The USD scheme provides traffic isolation between the customers of an ISP rather than between two classes.

- The primary service model in USD is proportional fairness rather than explicit bandwidth guarantee.

- The USD scheme enforces bandwidth allocation on the bottlenecks where the congestion takes place.

- The USD scheme does not require admission control at the edges of the network and it also works well with reverse traffic.

The USD scheme has two important components:

- User. A user is the basic entity to which the bandwidth is allocated. The term "user" refers to the party with whom an ISP enters a service contract and the entity that pays for the ISP's service. It is important to note that a user is not necessarily an end user; it can be a network or a group of networks.

- Each user is assigned a number called "share" based on how much a user has paid for the service. The share is used for determining how much bandwidth a user is allocated to. Under congestion, the share is used as the "weight" in the allocation of bandwidth.

The USD bandwidth allocation is carried out in the following steps:

- At the time when a user subscribes to its ISP for Internet services, the ISP and the user agree on the share for the user based on the user's requirements and how much it is willing to pay. The share may change each time when the user changes its service contract.

- At any time instance and any point inside the ISP's domain, the ISP con provide two guarantees to the user. First, a user will have a minimum mount of guaranteed bandwidth anywhere inside the ISP (the worst-case guarantee). And at any time instance, the amount of bandwidth allocated to a user is 
proportional to its share among all the active competing traffic on any links (proportional guarantee).

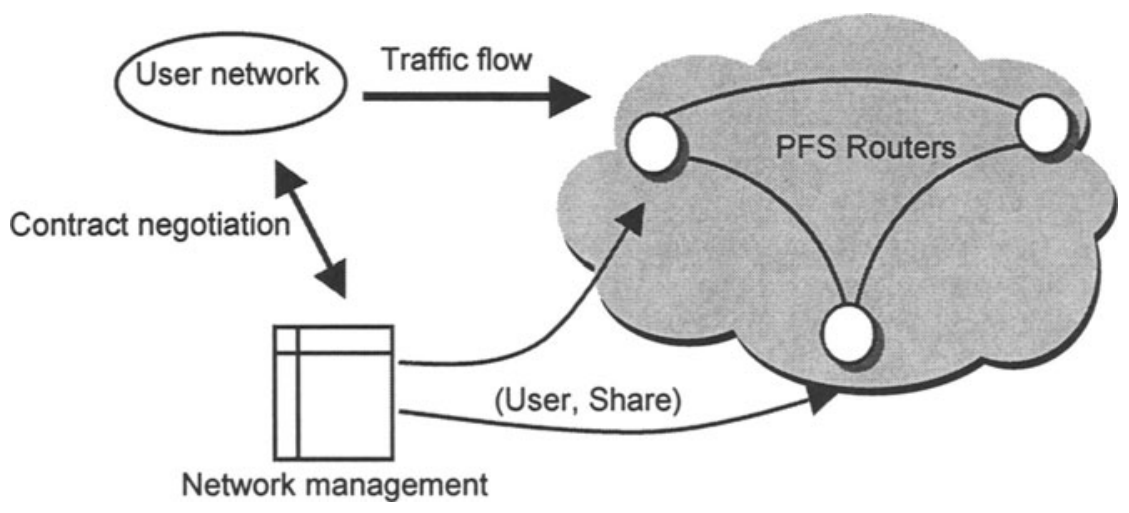

Figure 1: User-Share-Differentiation Information Flows

- The user and its corresponding share are distributed to some or all routers inside the ISP through some network management protocol such SNMP or other similar protocols.

- The USD allocation is policed with a scheduler that supports proportional fair sharing (PFS) and is activated whenever a queue builds up.

We now discuss the key design decisions behind the USD scheme.

\subsection{Flexible Control Granularity}

One of the main issues in any resource allocation is the granularity of the control. The finer granularity offers better control of resources but also brings the associated complexity in the setup of the state and classification of packets. Part of the problem with the EEPS model is its 5-tuple fine granularity and the signaling requirement that comes with it. On the other hand, the granularity decides the minimum level of control one can excise, and the level of traffic isolation can be supported. Therefore, it is an important engineering decision that has to be made.

In USD, we follow the natural administrative boundaries in the customer-ISP contractual relationship and introduce the user as the basic unit that defines control granularity. All traffic originated from or destined to a user is aggregated into a single flow, and the ISP provides protection for a user's traffic from other competing users. Within traffic of a single user, it is up to the user to decide how the bandwidth is used internally. The definition of user is flexible to allow variable granularity to meet different requirements. A user is an individual host identified by its IP address or it can also be a network identified by the network prefix, and an ISP identified by its prefixes. 
As the per-user granularity provides full traffic isolation between users, it takes away the incentives for misbehaving. If a misbehaving user ignores the congestion signal, and continues to send traffic at unsustainable rates, it can only waste the bandwidth that the user is allocated and cause its own packets to be dropped. We believe that once the traffic isolation is provided inside the network, users will start to deploy intelligent control congestion mechanisms for their own good.

\subsection{Scalable Aggregation}

The definition of user also determines the level of aggregation inside a network. Note that the Internet has a hierarchical structure of ISPs, from backbone ISPs to retail ISPs and to end users. USD follows the same structure and allows hierarchical aggregation.

When traffic goes across ISP boundaries, the level of traffic aggregation also changes accordingly. Within a user's immediate ISP to which the user has a direct contractual agreement, all traffic of the user is aggregated into a single flow. In the core backbone, the retail ISP has a contractual agreement with the backbone provider on the behalf of all users from the retail ISP. Thus, all traffic from and to the ISP is visible in the backbone as one flow. As the traffic moves from the sender toward the backbone, the level of aggregation increases while the level of control granularity decreases. Such variable levels of aggregation is essential for the scalability in the core backbone as the amount of state in a network only depends on the number of customers an ISP has direct contractual relationship.

When a packet traverses the network, the control policy can actually change as the user-ISP relationship changes. Take Fig. 2 for example. When a packet from user A enters ISP A, the packet is aggregated into the flow to or from user A and the bandwidth allocation within ISP A is determined by the share assigned to user A (the source address prefix). When the packet goes into ISP B, the destination address prefix becomes visible thus the allocation depends on the user B's contract with ISP B. Within the backbone, the packet is aggregated into the flow for ISP A or ISP B. Such variable level of aggregation ensures a great deal of scalability and is consistent with the contractual obligations for the parties along the path.

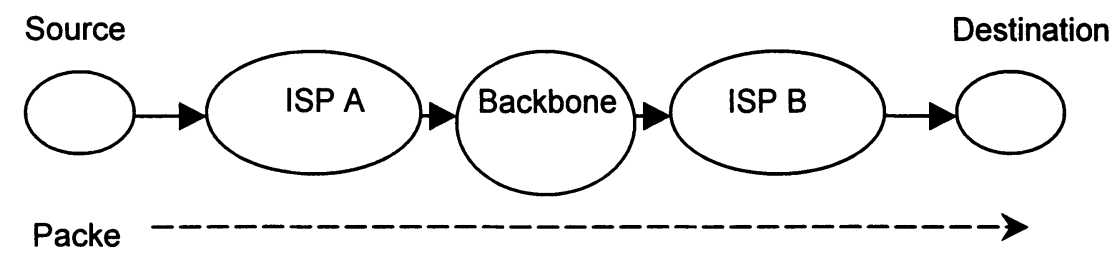

Figure 2: Variable Levels of Aggregation 


\subsection{Proportional Fair Sharing}

The per-user granularity allows traffic isolation between competing users. We can now move onto the policy for allocating bandwidth to multiple competing users.

In a commercial Internet, it is natural that the bandwidth allocation must be linked to how much a user has paid. This way, users can quantify the amount of services they get and thus are willing to pay more for better services. There are two basic approaches to achieve this goal. One can provide a user explicit bandwidth guarantee. Such guarantee would be easily to carry out if it is over a specific path, for example, a virtual leased line between two sites for a VPN application. However, to guarantee bandwidth anywhere in an ISP is much harder as one has to provision for the worst-case scenario. We believe that, for long-term bandwidth allocation anywhere in an ISP, guarantees for the relative fairness rather than the explicit amount of bandwidth is a more efficient option. In USD, the share reflects how big the slice of service that a user has paid for. When congestion occurs, the USD scheme allocates bandwidth to all active users at the bottleneck in proportional to the shares. For example, if user A and user B have shares of 5 and 10 respectively, user B will always get at least twice of what user A gets. If a user is consuming less bandwidth than it is allocated, the spare bandwidth is allocated in proportional to the other backlogged users. We call such a sharing model as "Proportional Fair Sharing". As we discuss in the next section, such sharing policy can be easily implemented with WFQ using the share as the weight.

Note that in the worst-case, the proportional allocation yields the same result as explicit allocation. For example, suppose that an ISP has 4 users A, B, C and D, sharing an access link of $30 \mathrm{Mbps}$. The agreed allocation is $4 \mathrm{Mbps}, 6 \mathrm{Mbps}, 8$ Mbps and $12 \mathrm{Mbps}$ respectively. This allocation can be described with the actual bandwidth, $4 \mathrm{Mbps}, 6 \mathrm{Mbps}, 8 \mathrm{Mbps}$ and $12 \mathrm{Mbps}$. Alternatively, the allocation can also be expressed with relative sharing, 2:3:4:6. When the 4 users are all active over the link, the bandwidth allocation is the same. However, the relative sharing has a number of advantages. First of all, it can guarantee the same minimum bandwidth allocation as an explicit allocation does. Second, it allows the bandwidth above the minimum to be shared in proportion to the minimum allocation. For example, suppose that user A and B in the previous example are not using their allocated bandwidth during a period. Now user $\mathrm{C}$ and $\mathrm{D}$ can share the extra bandwidth in proportion to their relative ratio. The final allocation to user $\mathrm{C}$ and $\mathrm{D}$ becomes $12 \mathrm{Mbps}$ and $18 \mathrm{Mbps}$. More importantly, the relative sharing representation works well with multiple bottlenecks with different bandwidth provision. For example, suppose the ISP of 4 users has another link with 600 Mbps bandwidth. The 4 users who have shares of 2, 3, 4, and 6 will have minimum guaranteed bandwidth automatically scaled up to $80 \mathrm{Mbps}, 120 \mathrm{Mbps}$, $160 \mathrm{Mbps}$ and $240 \mathrm{Mbps}$ respectively.

The relative sharing can be viewed as a flexible profile as it scales up and down according to the bandwidth available whilst guaranteeing the minimum bandwidth. In practice, the share can be defined in such a way that the share for a user can be easily derived from the minimum bandwidth allocated. For example, if we define 
the unit of share is $1 \mathrm{kbps}$, a user with $4 \mathrm{Mbps}$ minimum band-width has a share of 4000 .

\section{IMPLEMENTATION AND DEPLOYMENT}

Fig. 3 shows the block diagram for the implementation of the USD scheme in a router. The bandwidth allocation unit is similar to the IP lookup unit in IP routers and it consists of a bandwidth allocation table and a table lookup engine. The bandwidth allocation table is a list of user prefix and its associated share. The bandwidth allocation lookup engine does a longest prefix match with the source and destination of each packet to see if there is a match in the resource allocation table. If there is a match for either the source or the destination address, the associated share is used in the scheduler as the weight for bandwidth allocation. If both the source and destination match, this implies that both the sender and the destination are within the ISP. In such cases, the minimum of the two shares will be used for scheduling.

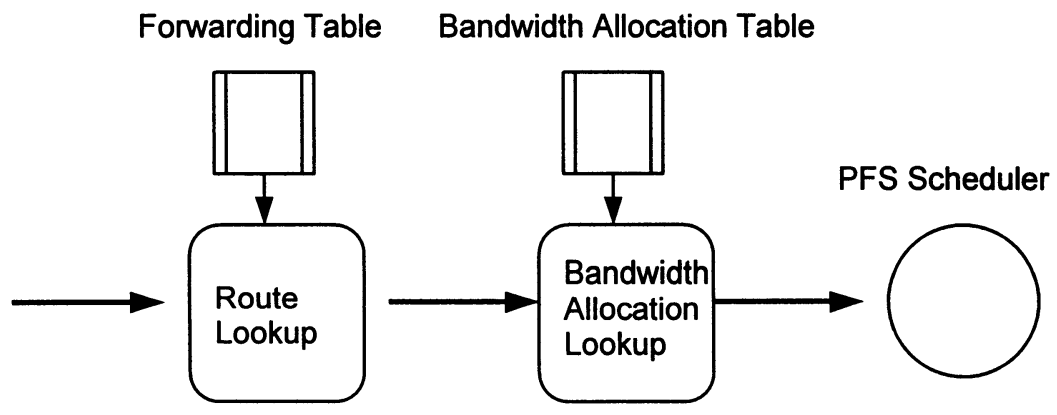

Figure 3: Block Diagram for the USD Implementation

To support USD, routers need to implement a scheduler that supports proportional fair sharing. There is a wide range of scheduling algorithms that meet such requirements. For example, Weighted Fair Queuing (WFQ) is such an algorithm that has been extensively in recent years (Parekh and Gallager 1992). Although the original WFQ is expensive to implement, several variations of WFQ have been proposed that support band-width sharing in similar fashion but are optimized for software and hardware implementation (Bennett and Zhang 1996, Shreedhar and Varghese 1995, Stiliadis 1996). Some of the algorithms can emulate WFQ closely with O(1) complexity (Shreedhar and Varghese 1995).

USD enforces bandwidth sharing locally on the bottleneck links. Thus it does not require any changes to the end systems and any admission control at the userISP boundaries. Consequently, USD can be deployed an incremental fashion. In fact, routers can be upgraded to support USD individually and each upgrade gives incremental improvement to the whole network. For example, when USD is 
installed on the router connected to the access link to the backbone, bandwidth allocation is enforced immediately for all traffic that is going through the access link. Moreover, USD only needs to be deployed at the points in the network that are heavily congested. Once bandwidth sharing is enforced at those points, other links may not require further policing.

\section{CONCLUSIONS}

In this paper, we examined the problems with the Premium service and Assured service proposals for the differentiated services, and presented the details of the USD scheme. We conclude that although the USD scheme requires more sophisticated support in the core network routers, it provides both minimum bandwidth guarantees and proportional fair sharing across the network and under various provisioning.

\section{REFERENCES}

Bennett, J. and Zhang, H (1996) WF2Q: Worst-case fair weighted fair queuing, IEEE INFOCOM'96.

Clark, D., Wroclawski, J. (1997) An Approach to Service Allocation in the Internet, Internet Draft, available at http://diffserv.lcs.mit.edu/Drafts/draftclark-diff-svc-alloc.00.txt.

Nichols, K., Jacobson, V. and Zhang, L. (1997) A Two-bit Differentiated Services Architecture for the Interne, Internet Draft, available at http://diffserv.lcs.mit.edu/Drafts/draft-nichols-diff-svc-arch-00.txt.

Parekh, A. K. and Gallager, G. R. (1992) A generalized processor sharing approach to flow control - the single node case, IEEE INFOCOM'92.

Shreedhar, M. and Varghese, G. (1995) Efficient Fair Queuing using Deficit Round Robin, ACM SIGCOMM'95.

Stiliadis, D. (1996) Traffic Scheduling in Packet Switched Networks: Analysis, Design and Implementation, Ph.D. Thesis, UC Santa Cruz, Ca, USA.

Wang, Z. (1997) User-Share Differentiation - scalable service allocation for the Internet, Internet Draft, available at http://diffserv.lcs.mit.edu/Drafts/draftwang-diff-serv-usd-00.txt.

Zhang, L., Deering, S., Estrin, D., Shenker, S. and Zappala, D. (1993) A New Resource Reservation Protocol, IEEE Network, 7 (5): 8-19. 